\title{
Sequential use of EGFR-tyrosine kinase inhibitors based upon EGFR mutation evolution achieves long-term control in a non-small cell lung cancer patient: a case report
}

\author{
Yueqiang Jiang ${ }^{1}$, Jun Zhang ${ }^{1}$, Xudong Jiang ${ }^{1}$, Ling Cheng ${ }^{1}$, Xin Liao ${ }^{1}$, Yuanyuan Li $^{1}$, Min Zhang ${ }^{2}$, \\ Rongrong Chen ${ }^{2}$, Tiejun Yin $^{1 \wedge}$ \\ ${ }^{1}$ Department of Geriatrics, Tongji Hospital, Tongji Medical College, Huazhong University of Science and Technology, Wuhan, China; ${ }^{2}$ Geneplus- \\ Beijing, Beijing, China \\ Correspondence to: Tiejun Yin. Department of Geriatrics, Tongji Hospital, Tongji Medical College, Huazhong University of Science and Technology, \\ Jiefang road No.1095, Wuhan 430030, China. Email: yfoxme@163.com.
}

\begin{abstract}
Tyrosine kinase inhibitor (TKI) has greatly improved the survival of non-small cell lung cancer (NSCLC) with epidermal growth factor receptor (EGFR)-TKI sensitive mutations. However, TKI resistance constantly occur, although multiple lines of different generations of TKIs are adopted during the progression. For example, in the case when T790M, which is the most common resistance mechanism of first generation TKIs, occurs, alteration to osimertinib (the third generation TKI) could always be effective. Unfortunately, some cases gradually become resistant even to osimertinib leaving limited therapy choice for clinical practitioners. Few cases have been reported in the situation after EGFR tertiary mutations occurred, such as C797S, G724S, etc. Herein, we report the first clinical evidence that sequential therapy with erlotinib, osimertinib, afatinib plus endostar, brigatinib plus cetuximab, almonertinib, almonertinib plus afatinib achieved long-term control in a NSCLC patient demonstrating EGFR 19Del/T790M/G724S/cis-C797S evolution in response to TKI treatment. EGFR targeted therapy introduced successful management for more than 36 months until now. ctDNA NGS was performed at the time of important clinical event. The EGFR 19Del was discovered in October 2017, and erlotinib was administered for 10 months with PR in the beginning. Then T790M was detected and osimertinib was used for 9 months with SD condition. Subsequently, EGFR G724S was identified in ctDNA with the remaining 19Del and loss of T790M. Afatinib plus endostar was administered and PR was achieved after 1.5 months. PD occurred 6 months later with the emergence of EGFR cis-C797S. Then brigatinib plus cetuximab was chosen and lasted for 4 months with the best response of SD. And then EGFR 19Del and T790M were still detected with loss of G724S and C797S. Almonertinib, another third-generation TKI, was administered for 3 months with SD condition. Finally, 19Del/T790M/G724S/cis-C797S recurred, and whole dose of almonertinib plus afatinib was prescribed until now with a PR at 2 months until now. The side effect was acceptable during the whole period of therapies. Plasma ctDNA NGS provided information of EGFR mutation evolution and inform appropriate therapy regimen during the progression.
\end{abstract}

Keywords: Non-small cell lung cancer (NSCLC); osimertinib resistance; EGFR evolution; G724S; C797S; case report

Submitted Jul 24, 2020. Accepted for publication Nov 12, 2020.

doi: 10.21037/apm-20-1477

View this article at: http://dx.doi.org/10.21037/apm-20-1477

^ ORCID: 0000-0002-1549-0829. 


\section{Introduction}

Epidermal growth factor receptor (EGFR) tyrosine kinase inhibitor (TKI) has greatly improved the survival of non-small cell lung cancer (NSCLC) with EGFR-TKI sensitive mutations compared to conventional standard platinum-based chemotherapy. After resistance to first generation TKI, most EGFR-TKI sensitive NSCLC develop secondary T790M mutation. Osimertinib, a third generation TKI, is highly effective in patients carrying primary EGFR-TKI sensitive mutations (such as exon 19 deletion (19Del) or L858R mutation) or T790M, a typical secondary mutation. However, resistance to osimertinib is still a huge challenge in clinic. The most common EGFR-dependent resistance mechanism to osimertinib is tertiary $E G F R$ mutations, such as C797S, L792H, L718Q, G724S, etc. (1). Few cases and no standard therapy have been reported in the situation after tertiary EGFR mutations occurred. Herein, we report a case of lung adenocarcinoma patient with EGFR mutation evolution involving 19Del, T790M, G724S and C797S, who benefited from sequential erlotinib, osimertinib, afatinib, brigatinib plus cetuximab, almonertinib, almonertinib plus afatinib therapy based on next-generation sequencing (NGS) results. We present the following article in accordance with the CARE reporting checklist (available at http://dx.doi. org/10.21037/apm-20-1477).

\section{Case presentation}

In December 2009, a 48-year-old male Chinese patient with smoking history was diagnosed with primary lung carcinoma by computed tomography (CT) screening with two lesions present in left lower and right lower lobes respectively. The diagnosis was further corroborated by pathological results after radically heterochronic surgery (both lesions are primary; left: poorly differentiated adenocarcinoma, pT1N0M0; right: papillary adenocarcinoma, pT2N0M0) in February and June 2010, respectively. No adjuvant chemotherapy or radiotherapy was performed after surgery. After follow-up for 5 years, multiple metastatic sites including bone, lung, pleura, and a back mass were detected) by positron emission tomography (PET)/CT in June 2015. Four cycles of chemotherapy with a regimen of pemetrexed plus carboplatin was adopted and resulted in a partial response (PR). Then the patient received Chinese medical herbs until June 2017 when new symptoms of severe headache and vomiting occurred. New cerebral and pituitary metastasis were detected by magnetic resonance imaging (MRI). Additionally, PET-CT revealed disease recurrence on cerebral, pituitary, and back mass. After whole brain radiotherapy with dose of 30Gy per 10 fractions, back mass was biopsied and metastasis from the lung adenocarcinoma was confirmed by pathological results. Further NGS analysis found EGFR 19Del mutation (allelic fraction, $\mathrm{AF}=25.2 \%$ ) in October 2017 (Figure 1).

Indicated by the genomic findings, erlotinib $(150 \mathrm{mg}$ per day) was prescribed, and the patient experienced partial response (PR) with a progression-free survival (PFS) of 10 months without obvious side effect. After progression disease (PD) in August 2018, plasma ctDNA sequencing suggested occurrence of EGFR T790M mutation $(\mathrm{AF}=1 \%)$ suggesting erlotinib resistance. The patient thereby received osimertinib ( $80 \mathrm{mg}$ per day) for 9 months without obvious adverse reactions and reached stable disease (SD) in the beginning. During osimertinib therapy, ctDNA analysis by NGS indicated the missing of T790M and recurrence of 19Del, alerting the potential resistance of osimertinib in February 2019, when pulmonary CT still showed SD.

In May 2019, pulmonary CT scanning suggested another PD again indicated by overgrowth of back mass. NGS-based ctDNA analysis found EGFR G724S mutation (AF $=14.9 \%)$ accompanied by 19Del with missing of T790M, and no other actionable mutations were detected. Afatinib (40 mg per day) combined with endostar (anti-angiogenic drug, $15 \mathrm{mg}$ per day for 2 weeks in every 3 weeks) was prescribed, and PR was achieved 1.5 months after initial treatment (Figure 2). In July 2019, ctDNA analysis indicated decreased AF of EGFR G724S mutation, suggesting that disease was under control. Afatinib plus endostar treatment lasted for 6 months with grade 1/2 skin rash, and PD occurred in December 2019.

Unexpectedly, EGFR C797S (AF =1\%) was identified in cis with T790M, and $\mathrm{G} 724 \mathrm{~S}(\mathrm{AF}=0.3 \%)$ still existed with lower frequency in plasma (Figure 3). Brigatinib (90 $\mathrm{mg}$ per day) plus cetuximab $\left(260 \mathrm{mg} / \mathrm{m}^{2}\right.$ per 2 weeks), which was reported previously in treatment of NSCLC (2), were then administered for 4 months with adverse event of grade 2 skin rash. SD was achieved 2 months post-treatment, however, PD 2 months later (Figure 2). Then sequencing from plasma revealed missing of EGFR C797S and G724S and occurrence of 19Del and T790M in April 2020. Almonertinib (110 mg per day), another third-generation TKI, was administered for 3 months and conferred a SD condition with no obvious side effect. However, in July 2020, EGFR 19Del, T790M, C797S and G724S were all detected in plasma ctDNA, with the highest $\mathrm{AF}$ present in G724S. Then almonertinib (110 mg per day) 


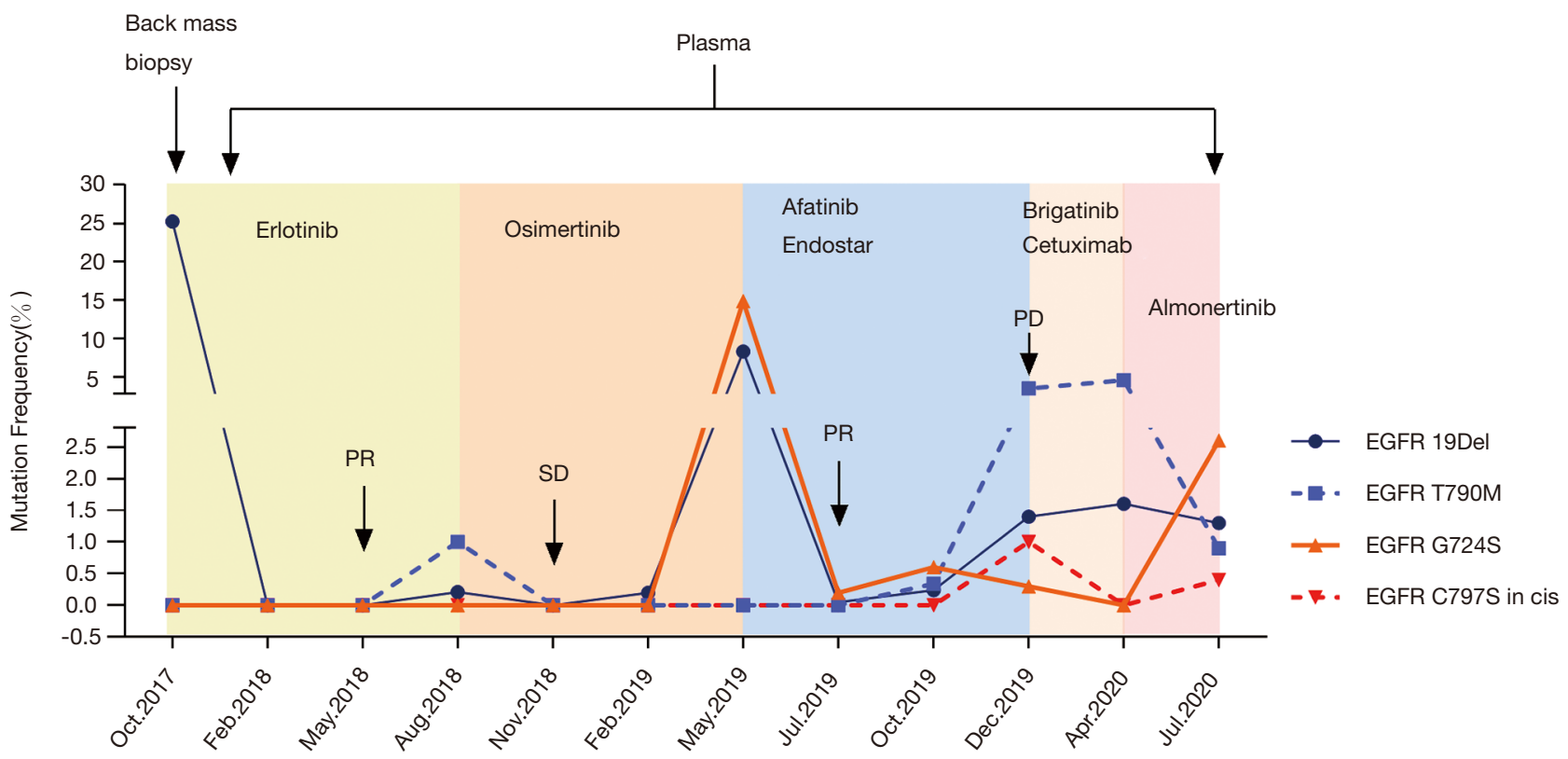

Figure 1 Dynamic monitoring of EGFR mutations during treatment with erlotinib, osimertinib, afatinib plus endostar, brigatinib plus cetuximab, almonertinib. PR, partial response; PD, progressive disease; SD, stable disease.

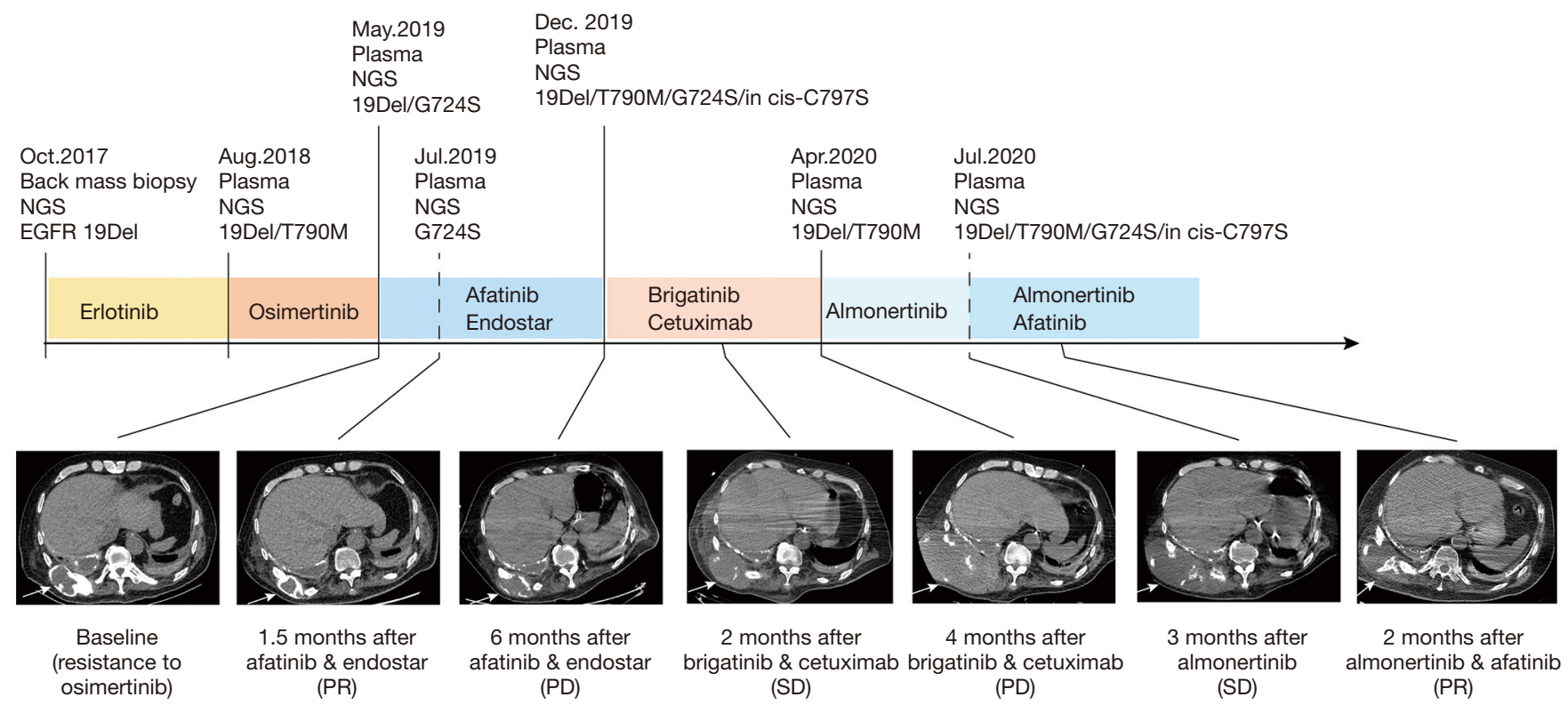

Figure 2 Clinical responses to afatinib plus endostar, brigatinib plus cetuximab, almonertinib, almonertinib plus afatinib (white arrow). The patient achieved a PR after 1.5 months treatment with afatinib plus endostar, and experienced PD after 6 months. Then the patient achieved a SD after 2 months treatment with brigatinib plus cetuximab, and experienced PD after 4 months. The best response was SD during almonertinib. And PR was achieved after 2 months treatment with almonertinib plus afatinib. NGS, next-generation sequencing; PR, partial response; $\mathrm{PD}$, progressive disease; $\mathrm{SD}$, stable disease. 

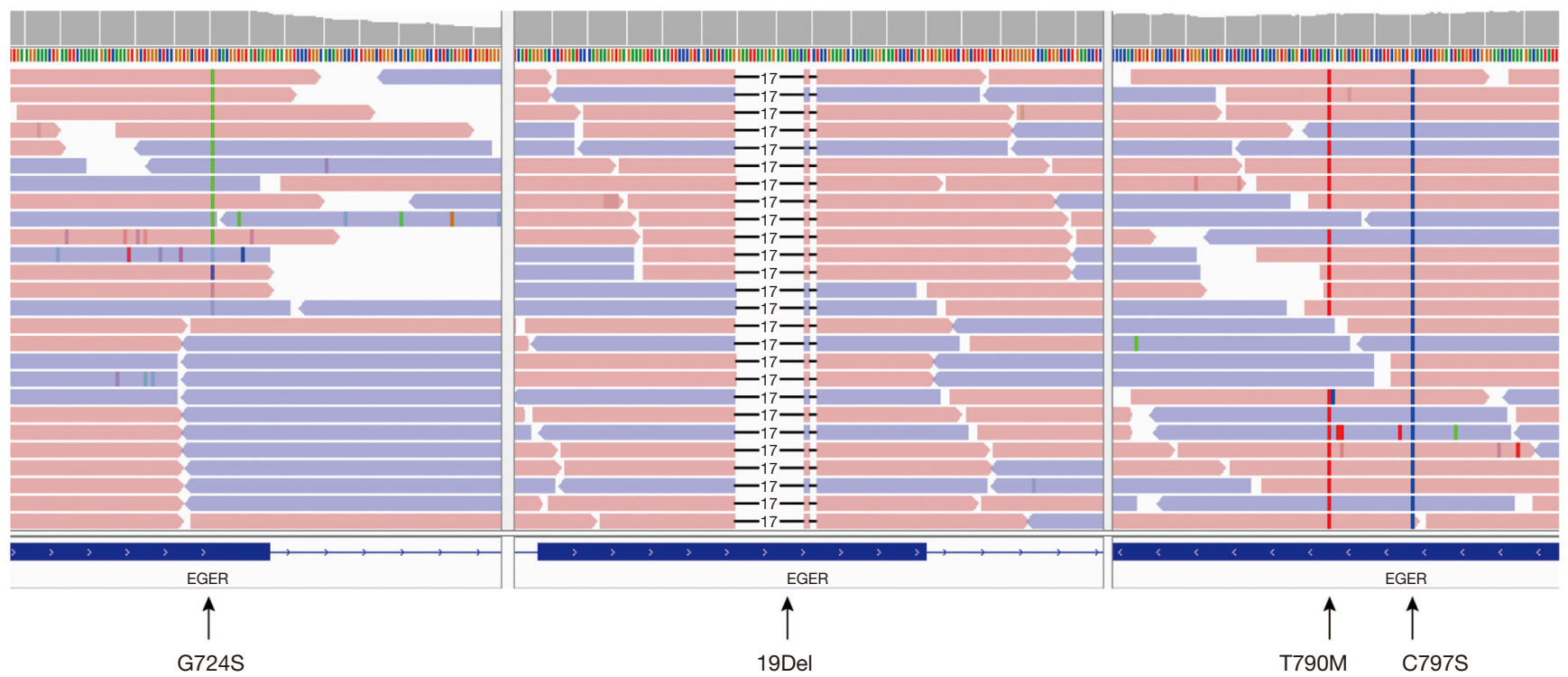

Figure 3 ctDNA sequencing reads of EGFR 19del/T790M/G724S/cis-C797S are shown by the Integrative Genomics Viewer (IGV).

plus afatinib (30 $\mathrm{mg}$ per day initially and then $40 \mathrm{mg}$ per day) were prescribed and PR was achieved 2 months later. And fortunately, only grade 2 skin rash and no interstitial lung diseases occurred until now. The overall EGFR mutation evolution is shown in Figure 4.

All procedures performed in this study involving human participants were in accordance with the ethical standards of the institutional and/or national research committee(s) and with the Helsinki Declaration (as revised in 2013). Written informed consent was obtained from the patient.

\section{Discussion}

TKIs provides a hopeful therapy choice for EGFR-mutant NSCLC patients. To overcome the resistance, the third generation of EGFR TKIs (such as osimertinib or almonertinib) have emerged. However, even in the case treated by multiple lines across different generations of TKIs, resistance still occurred to hinder the treatments efficacy. C797S is the most common tertiary EGFR mutation, which accounts for $15 \%$ of osimertinib resistance (1). G724S, another uncommon acquired tertiary mutation resistant to osimertinib, was thought to be only found in initial 19del mutant NSCLC but not in those withL858R (3-7). In our case, G724S displayed earlier than C797S and the latter worked as the resistance mechanism of the former. It has been reported in vitro that $\mathrm{G} 724 \mathrm{~S}$ could reduce osimertinib binding affinity in the context of exon 19 deletion, without affecting the affinity of L858R mutation. Structural analysis indicated that G724S may induce a conformation of the glycine-rich loop, which was incompatible with the binding of osimertinib and first-generation TKIs, while afatinib could retain kinase affinity and overcome G724S mediated resistance $(3,6)$.

Currently, there is no standard treatment for EGFR G724S mutation. Zhang et al. reported a NSCLC patient treated with osimertinib plus gefitinib after EGFR G724S mutation, but the combination therapy was only effective for 2 months and PD occurred soon. It has also been reported by Peled et al. that osimertinib plus low dose afatinib (20 $\mathrm{mg}$ per day) as an approach for EGFR G724S mutation with only grade 2 skin toxicity; however, the combination therapy only sustain for 3 months with liver PD (8). In other cases with acquired EGFR G724S mutation, efficacy of chemotherapy or immune checkpoint inhibitors was poor (4). Considering the application of afatinib in uncommon EGFR mutations, it is reasonable to suggest that sufficient dose of afatinib could serve as the basal treatment of G724S mutation. And in our case, afatinib plus endostar was chosen as the third-line treatment when G724S was detected. Combined therapy achieved PR in the beginning, and a PFS of 6 months with acceptable adverse event, which was proved to be an effective therapy scheme.

Unexpectedly, C797S mutation in cis with T790M, was detected in plasma ctDNA after resistance to afatinib plus endostar. Afatinib efficacy on C797S alone is still controversial. 


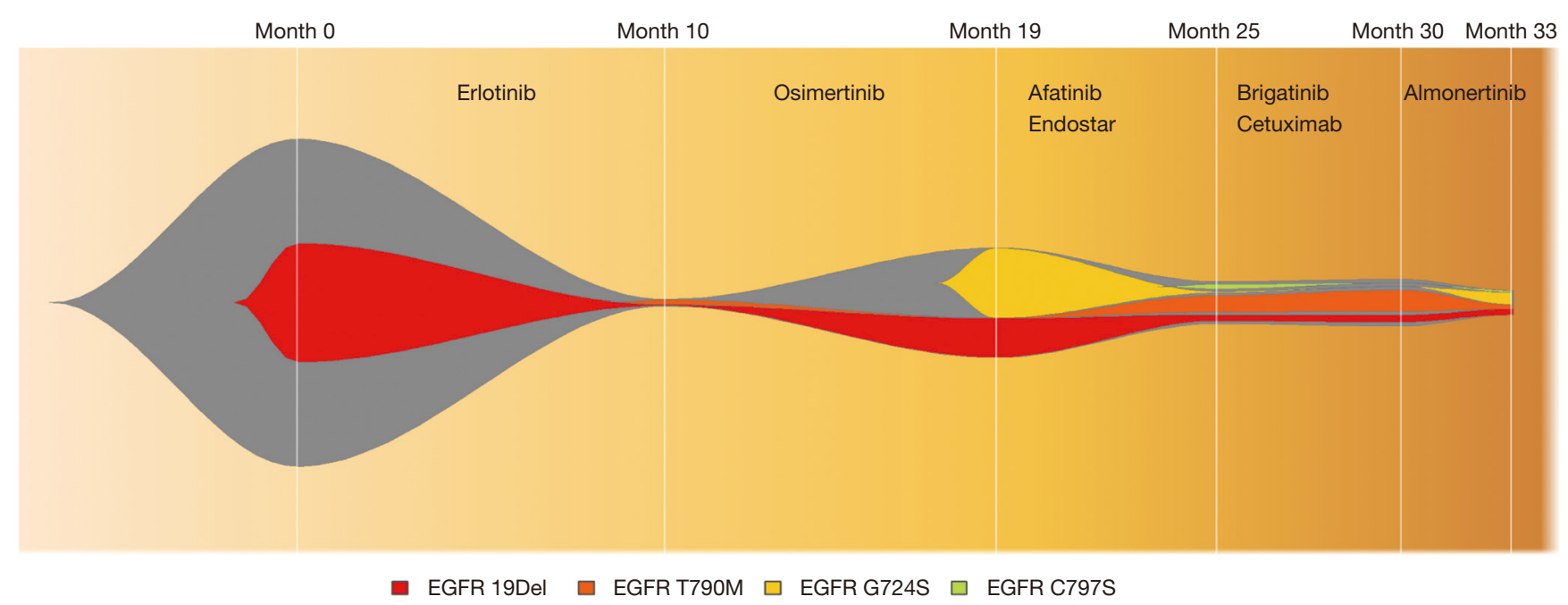

Figure 4 Schematic diagram of EGFR mutation evolution. The patient carried EGFR 19Del at the disease recurrence, and T790M appeared with erlotinib resistance. After osimertinib treatment, G724S was detected, and the therapy was switched to afatinib plus endostar. During disease progression, C797S in cis with T790M was identified, and the patient received brigatinib plus cetuximab. When disease progressed again, G724S and C797S disappeared, but 19Del and T790M still existed. Almonertinib, another third generation EGFR-TKI was administered. Three months later, G724S and C797S were detected again and afatinib was added.

NSCLC cell lines which expressed EGFR 19Del/C797S or C797S alone remained sensitive to afatinib (9). However, C797S could also be identified after afatinib therapy in patients without it previously, and may have less contribution to afatinib resistance than T790M (10). Cases have been reported that brigatinib plus cetuximab were effective which could overcome cis-C797S mutation (2). Thereby, brigatinib plus cetuximab was administered when 19Del/T790M/G724S/ cis-C797S were detected, and lasted for 4 months with weak efficacy. It is noteworthy that G724S/C797S disappeared and 19Del/T790M still existed after brigatinib plus cetuximab got resisted, which created an opportunity for the prescription of another third-generation TKI, almonertinib. However, efficacy of almonertinib only sustained for 3 months, and 19Del/T790M/G724S/cis-C797S recurred; due to the weak efficacy of brigatinib plus cetuximab in this situation and afatinib may work on C797S and G724S, whole dose of almonertinib plus afatinib was prescribed, which showed ideal control of disease (PR) until now.

Different from another case, in which KRAS was the subsequent resistance mechanism after EGFR L718Q, another osimertinib resistant mutation which is sensitive to afatinib (11), our work showed a case with long period of EGFR 19Del/T790M/G724S/cis-C797S evolution and successful sequential therapy using different generations of
TKIs. However, there are also limitations in our work. The dose of TKIs in our case may not be generalized; whole dose of almonertinib (110 mg per day) plus afatinib (40 mg per day) could betoxic, although it showed no severe side effect in our case. Therefore, we could not guarantee that whole dose of two kinds of TKIs at the same time could be applicable to other cases.

In a nutshell, this study reports the first clinical evidence that sequential therapy of erlotinib, osimertinib, afatinib plus endostar, brigatinib plus cetuximab, almonertinib, almonertinib plus afatinib in an advanced NSCLC patient with EGFR 19Del/T790M/G724S/cis-C797S evolution. EGFR targeted therapy introduced successful whole management more than 36 months until now. During the course, plasma ctDNA NGS provides information of EGFR mutations evolution and inform precise therapy.

\section{Acknowledgments}

We owe thanks to the patient and his family. Funding: None.

\section{Footnote}

Reporting Checklist: The authors have completed the CARE 
reporting checklist. Available at http://dx.doi.org/10.21037/ apm-20-1477

Peer Review File: Available at http://dx.doi.org/10.21037/ apm-20-1477

Conflicts of Interest: All authors have completed the ICMJE uniform disclosure form (available at http:// dx.doi.org/10.21037/apm-20-1477). The authors have no conflicts of interest to declare.

Ethical Statement: The authors are accountable for all aspects of the work in ensuring that questions related to the accuracy or integrity of any part of the work are appropriately investigated and resolved. All procedures performed in studies involving human participants were in accordance with the ethical standards of the institutional and/or national research committee(s) and with the Helsinki Declaration (as revised in 2013). Written informed consent was obtained from the patient.

Open Access Statement: This is an Open Access article distributed in accordance with the Creative Commons Attribution-NonCommercial-NoDerivs 4.0 International License (CC BY-NC-ND 4.0), which permits the noncommercial replication and distribution of the article with the strict proviso that no changes or edits are made and the original work is properly cited (including links to both the formal publication through the relevant DOI and the license). See: https://creativecommons.org/licenses/by-nc-nd/4.0/.

\section{References}

1. Oxnard GR, Hu Y, Mileham KF, et al. Assessment of resistance mechanisms and clinical implications in patients with EGFR T790M-positive lung cancer and acquired resistance to osimertinib. JAMA Oncol 2018;4:1527-34.

2. Wang Y, Yang N, Zhang Y, et al. Brief Report: Effective treatment of lung adenocarcinoma harboring EGFR-

Cite this article as: Jiang Y, Zhang J, Jiang X, Cheng L, Liao X, Li Y, Zhang M, Chen R, Yin T. Sequential use of EGFRtyrosine kinase inhibitors based upon EGFR mutation evolution achieves long-term control in a non-small cell lung cancer patient: a case report. Ann Palliat Med 2021;10(6):7051-7056. doi: 10.21037/apm-20-1477 activating mutation/T790M/cis-C797S triple mutations by brigatinib and cetuximab combination therapy. Comp Biochem Physiol A Mol Integr Physiol 2002;132:177-84.

3. Brown BP, Zhang YK, Westover D, et al. On-target Resistance to the Mutant-Selective EGFR Inhibitor Osimertinib Can Develop in an Allele-Specific Manner Dependent on the Original EGFR-Activating Mutation. Clin Cancer Res 2019;25:3341-51.

4. Oztan A, Fischer S, Schrock A, et al. Emergence of EGFR G724S mutation in EGFR-mutant lung adenocarcinoma post progression on osimertinib. Lung Cancer 2017;111:84-7.

5. Zhang Y, He B, Zhou D, et al. Newly emergent acquired EGFR exon 18 G724s mutation after resistance of a T790M specific EGFR inhibitor osimertinib in nonsmall-cell lung cancer: a case report. Onco Targets Ther 2019;12:51.

6. Fassunke J, Müller F, Keul M, et al. Overcoming EGFR G724S-mediated osimertinib resistance through unique binding characteristics of second-generation EGFR inhibitors. Nat Commun 2018;9:4655.

7. Li J, Wang Z, Groen HJ, et al. Uncommon EGFR G724S mutations arise in non-small-cell lung cancer patients with acquired resistance to first-generation EGFR-TKIs. Lung Cancer 2018;118:173-5.

8. Peled N, Roisman LC, Miron B, et al. Subclonal therapy by two EGFR TKIs guided by sequential plasma cellfree DNA in EGFR-mutated lung cancer. J Thorac Oncol 2017;12:e81-4.

9. Ercan D, Choi HG, Yun CH, et al. EGFR Mutations and Resistance to Irreversible Pyrimidine-Based EGFR Inhibitors. Clin Cancer Res 2015;21:3913-23.

10. Nakamura T, Nakashima C, Komiya K, et al. Mechanisms of acquired resistance to afatinib clarified with liquid biopsy. PLoS One 2018;13:e0209384.

11. Yang X, Huang C, Chen R, et al. Resolving resistance to osimertinib therapy with afatinib in an NSCLC patient with EGFR L718Q mutation. Clin Lung Cancer 2020;21:e258-60. 